\title{
Systematic method for solving strings having a symmetry in the space-time
}

\author{
Tatsuhiko Koike ${ }^{* a}$ Hideki Ishihara $^{b}$ and Hiroshi Kozaki ${ }^{c}$ \\ ${ }^{a}$ Department of Physics, Keio University, Yokohama 223-8522 Japan \\ ${ }^{b}$ Department of Mathematics and Physics, Graduate School of Science, Osaka City University, \\ Osaka 558-8585 Japan \\ ${ }^{c}$ Department of General Education, Ishikawa National College of Technology, Tsubata, Ishikawa \\ 929-0392 Japan \\ E-mail: koike@phys.keio.ac.jp ishihara@sci.osaka-cu.ac.jp \\ kozaki@ishikawa-nct.ac.jp
}

\begin{abstract}
In the presence of certain symmetry, called cohomogeneity-one symmetry, the equation of motion of an extended object in spacetime reduces to the problem of finding geodesics on a certain orbit space. We present a general method for obtaining solutions of such extented objects and classifying them. The classification is carried out in 4-dimensional Minkowski space and in 5dimensional anti-de Sitter space. The solvability condition is described in terms of Killing vectors and Killing tensors which mutually commute. In 4-dimensional Minkowski space, it is found that all types of cohomogeneity-one strings are solvable. In the case of 5-dimensional de Sitter space, there are not enough number of commuting Killing vectors but that the problem reduces to that of finding curves on a two-dimensional surface.
\end{abstract}

Black Holes in General Relativity and String Theory

24-30 August, 2008

Veli Losinj, Croatia

\footnotetext{
* Speaker.
} 
Existence and dynamics of extended objects play important roles in various stages in cosmology. Examples of extended objects include topological defects, such as strings and membranes, and the Universe as a whole embedded in a higher-dimensional spacetime in the context of the brane-world universe models [1]. The trajectory of an extended object is determined by a partial differential equation (PDE). For example, a test string is described by the Nambu-Goto equation which is a PDE in two dimensions. Because the dynamics is more complicated than that of a particle, one usually cannot obtain general solutions. One way to find exact solutions is to assume symmetry. The simplest solutions to such a PDE are homogeneous ones, in which case the problem reduces to a set of algebraic equations. However, the solutions do not have much variety and the dynamics is trivial.

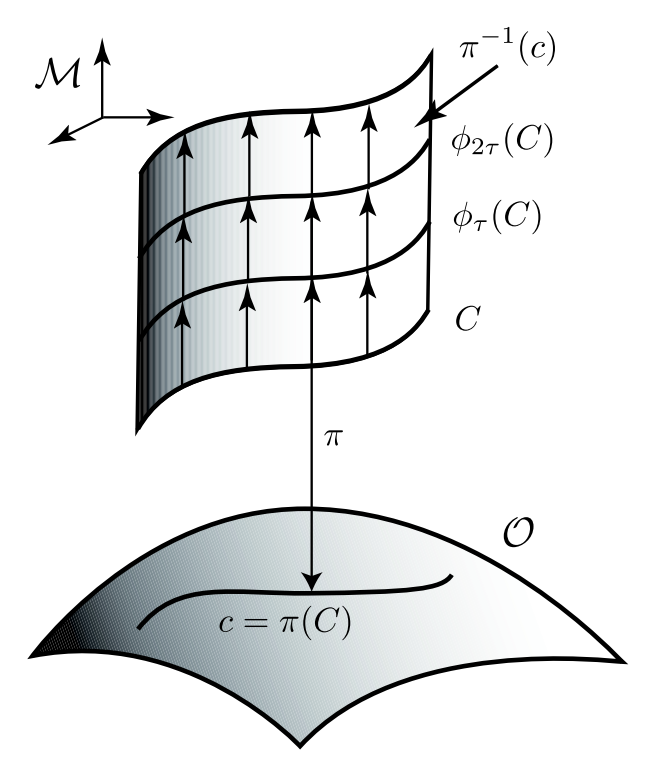

Figure 1: Solving a trajectory of the cohomogeneityone string reduces to finding a geodesic $c$ on the orbit space $(\mathscr{O},-f h)$.

the Nambu-Goto action

$$
S=\int_{S} d \sigma^{2} \sqrt{-\operatorname{det}\left(g_{a b} \frac{\partial x^{a}}{\partial \sigma^{\alpha}} \frac{\partial x^{b}}{\partial \sigma^{\beta}}\right)} .
$$

For a cohomogeneity-one string, one can carry out the integration in the direction of the Killing vector $\xi=\partial_{\tau}$ to obtain

$$
S=\int_{c} d \sigma \sqrt{-f h_{a b} \dot{x}^{a} \dot{x}^{b}}
$$

where

$$
h_{a b}:=g_{a b}-\xi_{a} \xi_{b} / f, \quad f:=\xi^{a} \xi_{a}
$$

and an overdot denotes the differentiation by $\sigma$. The integration in (2) is over a curve $c$ on the orbit space $\mathscr{O}$, which is defined by identifying all the points on each Killing orbit $\mathscr{S}_{\sigma}$ in $\mathscr{M}$. Thus 
the problem of the string reduces to finding geodesics on the orbit space $\mathscr{O}$ with the metric $-f h$ (Fig. 11. The metric $h$ has the Euclidean signature if $\xi$ is timelike, i.e., if $f<0$, and has the Lorentzian signature if $\xi$ is spacelike, i.e., if $f>0$. In practice, it is often more convenient to deal with a modified action

$$
S=\int_{c} d \sigma\left(-\frac{1}{\alpha} f h_{a b} \dot{x}^{a} \dot{x}^{b}+\alpha\right)
$$

which derives the same geodesic equations as (2) and retains the invariance under reparametrization of $\sigma$. The variable $\alpha$ is the norm of the vector tangent to $c$.

The procedure above can be carried out once $\xi$ (or $K$ ) is given, but different Killing vector fields $\xi$ and $\xi^{\prime}$ may derive the physically equivalent solution of the string. This happens when there is an isometry $\Phi$ on $\mathscr{M}$ such that

$$
\xi^{\prime}=\Phi_{*} \xi
$$

In algebraic terms, the complete classification of cohomogeneity-one string is to determine $\mathfrak{g} / \mathrm{Ad}_{\mathfrak{g}}$ where $\mathfrak{g}$ denotes the Lie algebra of the isometry group of $\mathscr{M}$, i.e. the set of Killing vector fields on $\mathscr{M}$. The classification is done for 4-dimensional Minkowski space $E^{3,1}$ (Table 11] [2] and for 5-

\begin{tabular}{cc}
\hline Type & Killing vector $\xi$ \\
\hline I & $a P_{t}+b J_{z}$ \\
II & $a P_{t}+b P_{z}$ \\
III & $a P_{z}+b J_{z}$ \\
IV & $a P_{z}+J_{z}+K_{y}$ \\
V & $a P_{z}+b K_{y}$ \\
VI & $a P_{x}+J_{z}+b K_{y}$ \\
VII & $J_{z}+a K_{z}(a \neq 0)$ \\
\hline
\end{tabular}

Table 1: The classification of the cohomogenetyone strings in the 4-dimensional Minkowski space by the Killing vector $\xi$ along the world sheet. dimensional anti-de Sitter space $A d S^{5}$ (Table 2) [3].

We have explained how to classify the cohomogeneity-one strings and how to reduce the problem of finding their trajectories to a set of ODEs. The obtained ODEs can always be solved numerically, but they are not always solved analytically. Thus the problem of solvability is of interest. The simplest sufficient condition for the solvability of the geodesics on $(\mathscr{O},-f h)$ is that there are $(\operatorname{dim} \mathscr{O}-1)$ independent, mutually commuting Killing vector fields on $(\mathscr{O},-f h)$. In order to determine the isometry group of $(\mathscr{O},-f h)$, it is important to notice that the orbit space $(\mathscr{O},-f h)$

\begin{tabular}{cc}
\hline Type & Killing vector $\xi$ \\
\hline$(4 \mid 0)$ & $K_{x}+\widetilde{K}_{y}+J_{x y}+L+2\left(J_{y z}+K_{z}\right)$ \\
$( \pm 3, \mp 1 \mid 0)$ & $K_{x}+\widetilde{K}_{y}+J_{y z} \mp J_{x w}+a\left(J_{x y}-L \pm J_{z w}\right)$ \\
$(2,2 \mid 0)$ & $K_{x}+L+a J_{y z}$ \\
$(2,-2 \mid 0)$ & $K_{x}+J_{x y}+a J_{z w}$ \\
$(2,1,1 \mid 0)$ & $K_{x}+\widetilde{K}_{y}+J_{x y}+L+a J_{z w}+b\left(J_{x y}-L\right)$ \\
$(1,1,1,1 \mid 0)$ & $a L+b J_{x y}+c J_{z w}$ \\
$(2 \mid 1)$ & $K_{x}+\widetilde{K}_{y}+L+J_{x y}+a J_{z w}+b\left(K_{y}+\widetilde{K}_{x}\right)$ \\
$(1,1 \mid 1)$ & $K_{x}+\widetilde{K}_{y}+a J_{z w}+b\left(L-J_{x y}\right)$ \\
$(0 \mid 2)$ & $K_{x}+J_{x y}+a \widetilde{K}_{z}(a \neq 0)$ \\
$(0 \mid 1,1)$ & $a K_{x}+b \widetilde{K}_{y}+c J_{z w} \quad(b \neq \pm a)$ \\
\hline
\end{tabular}

Table 2: The classification of cohomogeneity-one strings in 5-dimensional anti-de Sitter space. 
"inherits" some of the isometries from $(\mathscr{M}, g)$. Namely, if an isometry $\Phi$ on $\mathscr{M}$ commutes with $\xi$, one can define a map $\Phi^{\mathscr{O}}$ from $\mathscr{O}$ to itself by $\Phi^{\mathscr{O}}(x):=\pi \circ \Phi(p)$, where $\pi$ is the projection from $\mathscr{M}$ to $\mathscr{O}$ and $p$ is any point such that $\pi(p)=x$. It is easily shown that $\Phi^{\mathscr{O}}$ preserves $f$ as well as $h$ and thus is an isometry on $(\mathscr{O},-f h)$. Therefore the group formed by the isometries $\mathscr{M}$ which commutes with $\xi$ gives (a subset of) the isometry group of $(\mathscr{O},-f h)$. In the case of the Minkowski space $E^{3,1}$, this analysis shows that for all types of $\xi$ except Type VII, the geodesic on $(\mathscr{O},-f h)$ are solvable. However, an interesting fact is that the geodesics also turn out to be solvable for Type VII. Namely, one can show the existence of a nontrivial Killing tensor on $(\mathscr{O},-f h)$ which ensures the solvability [4]. We have performed the same analysis in for all the types in the case of $A d S^{5}$ found in Ref. [3]. It is found that for all the types there are two commuting Killing vector fields on $(\mathscr{O},-f h)$. This shows that the problem of solving cohomogeneity-one strings reduces to a problem of finding certain curves on a two-dimensional surface, but it is not known whether they are solvable. Existence or inexistence of nontrivial Killing tensors on $(\mathscr{O},-f h)$ is unknown. In Ref. [3], the solutions is given in the special case when there are more than two commuting Killing vector fields on $(\mathscr{O},-f h)$.

The strings solutions found in the procedure above are treated as test strings. It is interesting to include gravitational couplings with the background spacetime. Gravitational radiations emitted by those strings and the back reaction to the string are being calculated [5, 6].

\section{References}

[1] L. Randall and R. Sundrum, Phys. Rev. Lett. 83, (1999) 3370.

[2] H. Ishihara and H. Kozaki, Phys. Rev. D72, 061701(R) (2005).

[3] T. Koike, H. Kozaki and H. Ishihara, Phys. Rev. D77, 125003 (2008).

[4] H. Kozaki, T. Koike and H. Ishihara, in preparation.

[5] K. Ogawa, H. Ishihara, H. Kozaki and H. Nakano, arXiv:0811.2846.

[6] T. Suehiro and T. Koike, in preparation. 